\title{
NON-NEWTONIAN CONDUCTING FLUID FLOW AND HEAT TRANSFER DUE TO A ROTATING DISK
}

\author{
HAZEM A. ATTIA' and MOHAMED E. S. AHMED ${ }^{1}$
}

(Received 2 January, 2003; revised 24 October, 2004)

\begin{abstract}
The steady flow of an incompressible viscous non-Newtonian electrically conducting fluid and heat transfer due to the rotation of an infinite disk are studied considering the Hall effect. The effects of an externally applied uniform magnetic field, the Hall current, and the non-Newtonian fluid characteristics on the velocity and temperature distributions as well as the heat transfer are considered. Numerical solutions of the nonlinear equations which govern the magnetohydrodynamics (MHD) and energy transfer are obtained over the entire range of the physical parameters.
\end{abstract}

\section{Introduction}

The pioneering study of fluid flow due to an infinite rotating disk was carried out by von Karman in 1921 [21]. He gave a formulation of the problem and then introduced his famous transformations which reduced the governing partial differential equations to ordinary differential equations. Cochran [8] obtained asymptotic solutions for the steady hydrodynamic problem formulated by von Karman. Benton [7] improved Cochran's solutions and solved the unsteady problem. The hydromagnetic flow of a Newtonian conducting fluid above a rotating disk with and without the Hall effect has been considered by many authors $[1,4,6,10,11]$. In all of the above studies the fluid is assumed to be Newtonian. The steady flow of a non-Newtonian fluid due to a rotating disk with uniform suction was considered by Mithal [15]. The solutions obtained were valid for small values of the parameter which describes the non-Newtonian behaviour. Srivastava [18] extended the problem to the case where the flow is between two infinite disks; one of which is rotating and the other of which is at rest. The hydromagnetic flow of a conducting non-Newtonian fluid due to the uniform rotation of an infinite

\footnotetext{
'Dept. of Eng. Mathematics and Physics, Fac. of Eng., Cairo University (El-Fayoum Branch), Egypt; e-mail: ah1113@yahoo.com.

(C) Australian Mathematical Society 2004, Serial-fee code 1446-1811/04
} 
disk in the presence of a uniform magnetic field was studied by Andersson and de Korte [3] who assumed that Hall currents were negligible.

The problem of heat transfer from a rotating disk maintained at a constant temperature was first considered by Millsaps and Pohlhausen [14] for a variety of Prandtl numbers in the steady state. Sparrow and Gregg [17] studied the steady-state heat transfer from a rotating disk maintained at a constant temperature to fluids at any Prandtl number. Later, many authors studied the heat transfer near a rotating disk considering different thermal conditions $[5,12,13,16,20]$.

In the present work, the steady MHD laminar flow and heat transfer of a viscous, electrically conducting, incompressible and non- Newtonian Reiner-Rivlin fluid due to the uniform rotation of a disk of infinite extent is studied taking the Hall effect into consideration. An external uniform magnetic field is directed perpendicular to the disk and the induced magnetic field is neglected by assuming that the magnetic Reynolds number $R_{\mathrm{em}} \ll 1[9,19]$. The temperature of the disk is impulsively changed and then maintained at a constant value. The governing nonlinear differential equations are solved numerically using finite differences. The effects of the applied uniform magnetic field, the Hall current, and the characteristics of the non-Newtonian fluid on the unsteady flow and heat transfer are presented and discussed.

\section{Basic equations}

Let the disk lie in the plane $z=0$ and the space $z>0$ be equipped by a viscous, incompressible, conducting and non-Newtonian Reiner-Rivlin fluid. The disk is rotating with a constant angular velocity $\omega$ about the line $r=0$ and an external uniform magnetic field is applied perpendicular to the plane of the disk which has a constant magnetic flux density $\boldsymbol{B}_{0}$. The magnetic Reynolds number is assumed to be very small, so that the Hall effect cannot be neglected $[9,19]$. The fluid motion is governed by $[4,6,15]$

$$
\begin{aligned}
\frac{\partial u}{\partial r}+\frac{u}{r}+\frac{\partial w}{\partial z} & =0 \\
\rho\left(u \frac{\partial u}{\partial r}+w \frac{\partial u}{\partial z}-\frac{v^{2}}{r}\right)+\frac{\sigma B_{0}^{2}}{1+m^{2}}(u-m v) & =\frac{\partial \tau_{r}^{r}}{\partial r}+\frac{\partial \tau_{r}^{z}}{\partial z}+\frac{\tau_{r}^{r}-\tau_{\phi}^{\phi}}{r} \\
\rho\left(u \frac{\partial v}{\partial r}+w \frac{\partial v}{\partial z}+\frac{u v}{r}\right)+\frac{\sigma B_{0}^{2}}{1+m^{2}}(u+m v) & =\frac{\partial \tau_{\phi}^{r}}{\partial r}+\frac{\partial \tau_{\phi}^{z}}{\partial z}+\frac{2 \tau_{\phi}^{r}}{r} \\
\rho\left(u \frac{\partial w}{\partial r}+w \frac{\partial w}{\partial z}\right) & =\frac{\partial \tau_{z}^{r}}{\partial r}+\frac{\partial \tau_{z}^{z}}{\partial z}+\frac{\tau_{z}^{r}}{r}
\end{aligned}
$$

where $u, v, w$ are velocity components in the directions of increasing $r, \phi, z$ respectively, $\rho$ is the density of the fluid, $\sigma$ is the electrical conductivity of the fluid, 
$m\left(=\sigma \beta B_{0}\right)$ is the Hall parameter which can take positive or negative values and $\beta$ is the Hall factor $[4,6]$. Positive values of $m$ mean that $\boldsymbol{B}_{0}$ is upwards and the electrons of the conducting fluid gyrate in the same sense as the rotating disk. For negative values of $m, \boldsymbol{B}_{0}$ is downwards and the electrons gyrate in an opposite sense to the disk. The constitutive equation for the non- Newtonian Reiner-Rivlin fluid is given by $[15,18]$

$$
\tau_{j}^{i}=2 \mu e_{j}^{i}+2 \mu_{c} e_{k}^{i} e_{j}^{k}-p \delta_{j}^{i}, \quad e_{j}^{j}=0,
$$

where $p$ denotes the pressure, $\tau_{j}^{i}$ is the stress tensor, $e_{j}^{i}$ is the rate of strain tensor, $\mu$ is the coefficient of viscosity and $\mu_{c}=\alpha\left(c+\sum \sum e_{j}^{i} e_{j}^{i}\right) / 2$ is the coefficient of cross viscosity. Here $c$ is a constant and $\alpha$ is a sufficiently small constant. The Reiner-Rivlin model is a simple model which can provide some insight into predicting the flow characteristics and heat transfer performance for viscoelastic fluid above a rotating disk. The first term in the right-hand side of (2.5) represents the viscous property of the fluid and the third term represents the elastic property of the fluid. We introduce the von Karman transformations [21],

$$
u=r w F, \quad u=r w G, \quad w=\sqrt{\omega \nu} H, \quad z=\sqrt{\nu / \omega} \zeta, \quad p-p_{\infty}=-\rho \nu \omega P,
$$

where $\zeta$ is a non-dimensional distance measured along the axis of rotation, $F, G, H$ and $P$ are non-dimensional functions of $\zeta$, and $\nu$ is the kinematic viscosity of the fluid, $\nu=\mu / \rho$. We define the magnetic interaction number $\gamma$ by $\gamma=\sigma B_{0}^{2} / \rho \omega$ which represents the ratio between the magnetic force to the fluid inertia force. With these definitions, (2.1)-(2.5) take the form

$$
\begin{aligned}
H^{\prime}+2 F & =0, \\
F^{\prime \prime}-H F^{\prime}-F^{2}+G^{2}-\frac{\gamma(F-m G)}{1+m^{2}}-\frac{K}{2}\left(F^{2}+3 G^{2}+2 F F^{\prime \prime}\right) & =0, \\
G^{\prime \prime}-H G^{\prime}-2 F G-\frac{\gamma(G+m F)}{1+m^{2}}+K\left(F^{\prime} G^{\prime}-F G^{\prime \prime}\right) & =0, \\
H^{\prime \prime}-H H^{\prime}-\frac{7}{2} K H^{\prime} H^{\prime \prime}+P^{\prime} & =0,
\end{aligned}
$$

where the prime denotes differentiation with respect to $\zeta$ and $K$ is the parameter that describes the non-Newtonian behaviour, $K=\mu_{c} \omega / \mu$. The boundary conditions for the velocity problem are given by

$$
\begin{aligned}
& F(0)=0, \quad G(0)=1, \quad H(0)=0, \\
& F(\infty)=0, \quad G(\infty)=1, \quad P(\infty)=0 .
\end{aligned}
$$

Equation (2.10a) indicates the no-slip condition of a viscous flow applied at the surface of the disk. Far from the surface of the disk, all fluid velocities must vanish 
aside the induced axial component as indicated in (2.10b). The above system of (2.6)-(2.8) with the prescribed boundary conditions given by (2.10) are sufficient to solve for the three components of the flow velocity. Equation (2.9) can be used to solve for the pressure distribution if required.

Due to the difference in temperature between the wall and the ambient fluid, heat transfer takes place. The energy equation, neglecting the dissipation terms, takes the form [17]

$$
\rho c_{\rho}\left(u \frac{\partial T}{\partial r}+w \frac{\partial T}{\partial z}\right)-K \frac{\partial^{2} T}{\partial z^{2}}=0 .
$$

The boundary conditions for the energy problem are that the temperature, by continuity considerations, equals $T_{w}$ at the surface of the disk. At large distances from the disk, $T$ tends to $T_{\infty}$, where $T_{\infty}$ is the temperature of the ambient fluid.

In terms of the non-dimensional variable $\theta=\left(T-T_{\infty}\right) /\left(T_{w}-T_{\infty}\right)$ and using the von Karman transformations, (2.11) takes the form

$$
\theta^{\prime \prime}-\operatorname{Pr} H \theta^{\prime}=0,
$$

where $\operatorname{Pr}$ is the Prandtl number given by $\operatorname{Pr}=c_{p} \mu / k$. The initial and boundary conditions are expressed in terms of $\theta$ as

$$
\theta(0)=1, \quad \theta(\infty)=0 .
$$

The heat transfer from the disk surface to the fluid is computed by application of an Fourier's law

$$
q=-k(d T / d z)_{w} .
$$

Introducing the transformed variables, the expression for $q$ becomes

$$
q=-k\left(T_{w}-T_{\infty}\right) \sqrt{\frac{\omega}{v}} \theta^{\prime}(0) .
$$

By rephrasing the heat transfer results in terms of a Nusselt number defined as $N_{u}=$ $q \sqrt{\nu / \omega} / k\left(T_{w}-T_{\infty}\right)$, the last equation becomes

$$
N_{u}=-\theta^{\prime}(0)
$$

The system of non-linear equations (2.6)-(2.8) and (2.12) is solved numerically under the conditions given by (2.10) and (2.13) using a marching technique and by applying the Crank-Nicolson implicit method [2]. The resulting system of difference equations has to be solved in the infinite domain $0<\zeta<\infty$. A finite domain in 
the $\zeta$-direction can be used instead with $\zeta$ chosen large enough to ensure that the solutions are not affected by imposing the asymptotic conditions at a finite distance. The independence of the results from the length of the finite domain and the grid density was ensured and successfully checked by various trial and error numerical experimentations. Computations are carried out for $\zeta_{\infty}=10$ which is found adequate for the ranges of the parameters studied here. It should be pointed out that the steadystate solutions reported by Aboul-Hassan and Attia [1] may be reproduced by setting $K=0$ in the present results. Also, the results obtained here coincide with the steadystate results approached in [6] for the case of zero suction. These comparisons lend confidence to the correctness of the solutions presented in this paper.

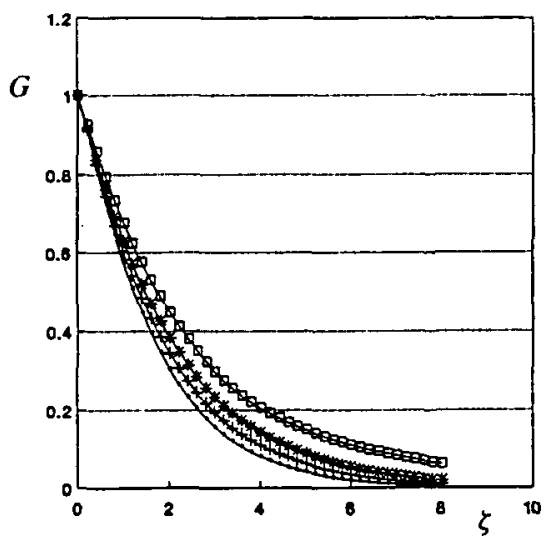

(a)

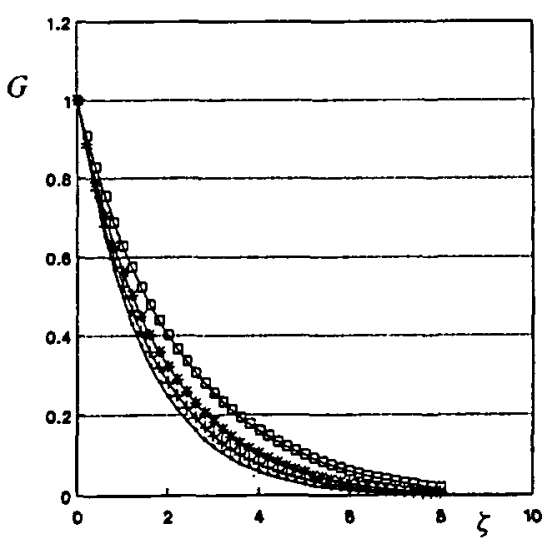

(c)

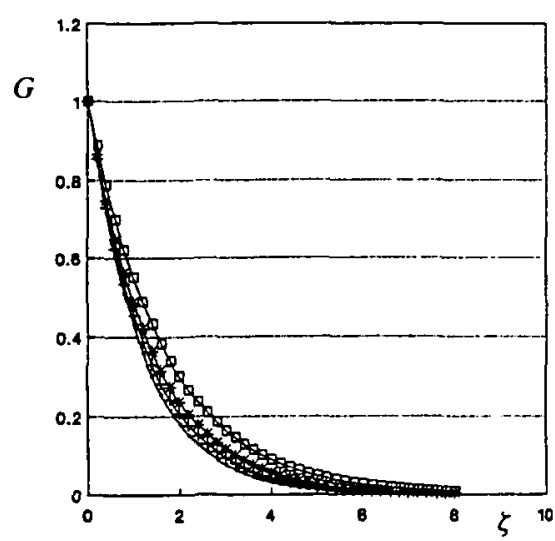

(b)



(d)

FIGURE 1. The steady-state profile of $G$ for various values of $K(\cdot K=0,+K=0.5, * K=1, \square K=2)$ and for (a) $\gamma=0$, (b) $\gamma=1, m=0$, (c) $\gamma=1, m=-0.5$ and (d) $\gamma=1, m=0.5$. 


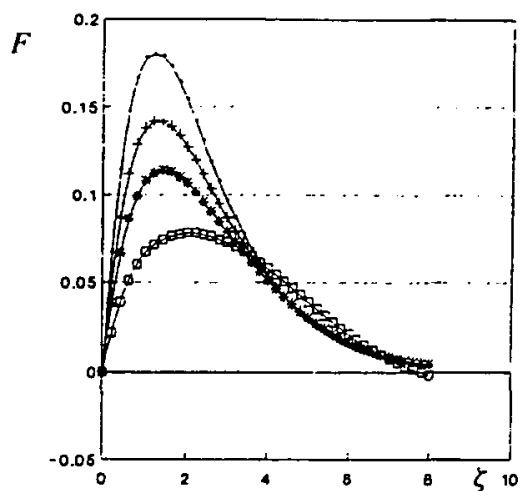

(a)

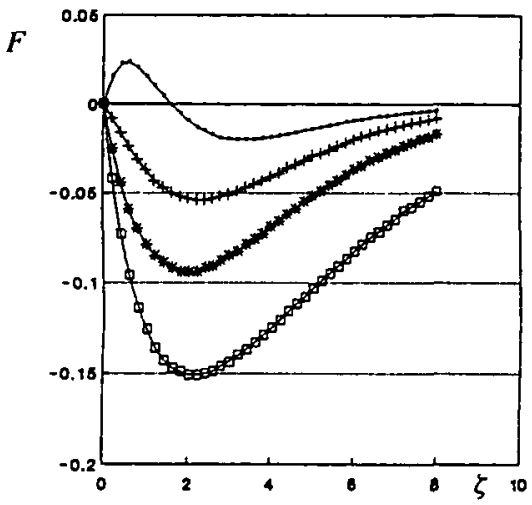

(c)



(b)

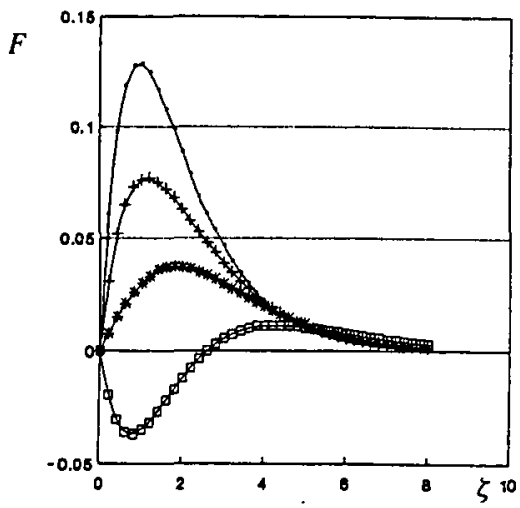

(d)

FIGURE 2. The steady-state profile of $F$ for various values of $K(\cdot K=0,+K=0.5, * K=1, \square K=2)$ and for (a) $\gamma=0$, (b) $\gamma=1, m=0$, (c) $\gamma=1, m=-0.5$ and (d) $\gamma=1, m=0.5$.

\section{Results and discussion}

The Hall parameter $m$ appears in the magnetic force terms and its contribution is proportional to $(F-m G) /\left(1+m^{2}\right)$ or $(G+m F) /\left(1+m^{2}\right)$. For small values of $m$, the effect of $m$ on the numerator is stronger than its effect on the denominator. A small positive value of $m$ decreases the magnetic damping on $F$ and increases the magnetic damping on $G$, thus increases $F$ and decreases both $G$ and $H$ (as follows from the continuity equation). A small negative value of $m$ decreases $F$ and increases both $G$ and $H$. For large positive values of $m$, the factor $(F-m G)$ may turn out to be negative and the magnetic field has a propelling effect on $F$, which may exceed its hydrodynamic value and thus the value of $H$ is below its hydrodynamic value. For such large values of $m$ the effect on $G$ is due mainly to the factor $1 /\left(1+m^{2}\right)$ 


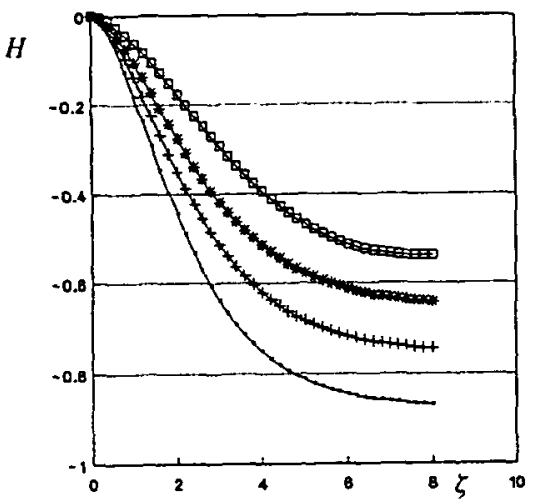

(a)

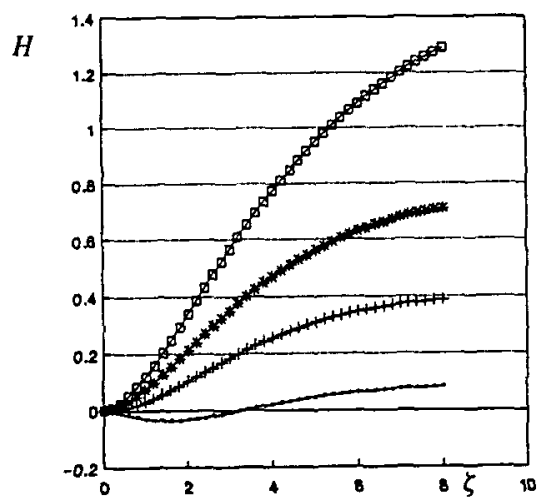

(c)

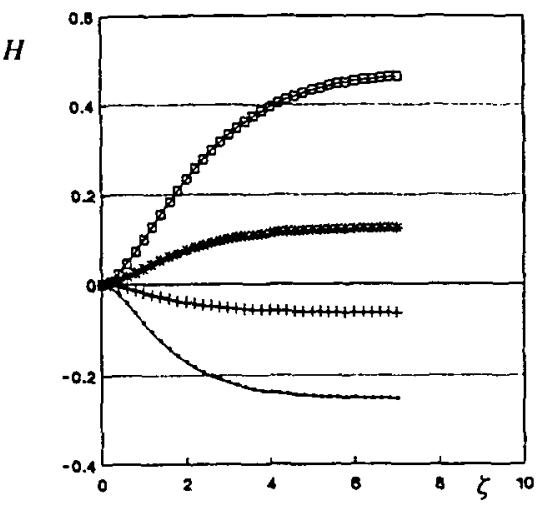

(b)



(d)

FIGURE 3. The steady-state profile of $H$ for various values of $K(\cdot K=0,+K=0.5, * K=1$, $\square K=2$ ) and for (a) $\gamma=0$, (b) $\gamma=1, m=0$, (c) $\gamma=1, m=-0.5$ and (d) $\gamma=1, m=0.5$.

which becomes very small and produces an increase in $G$. For large negative values of $m$ the argument is reversed. The magnetic damping on $F$ is reduced due to the decrease in $1 /\left(1+m^{2}\right)$. Thus $F$ increases but is still less than its hydrodynamic value, and consequently $H$ decreases but is more than its hydrodynamic value. The factor $(G+m F)$ may become negative and this pushes $G$ above its hydrodynamic value, and thus the magnetic field has a propelling effect on $G$. For very large positive or negative values of $m$ the magnetic force term decreases considerably and the limit $m=+\infty$ or $-\infty$ corresponds to the hydrodynamic limit.

Figure 1 presents the value of the azimuthal velocity component $G$ as a function of $\zeta$ for various values of the parameters $K, \gamma$ and $m$. Increasing the parameter $K$ increases $G$ for all values of $\gamma$ and $m$. Comparing Figures 1 (a) and (b) indicates that increasing $\gamma$ decreases $G$ for all values of $K$ and its effect becomes more pronounced 


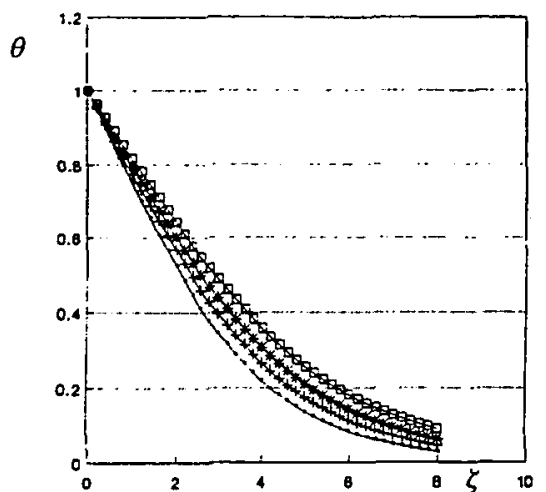

(a)

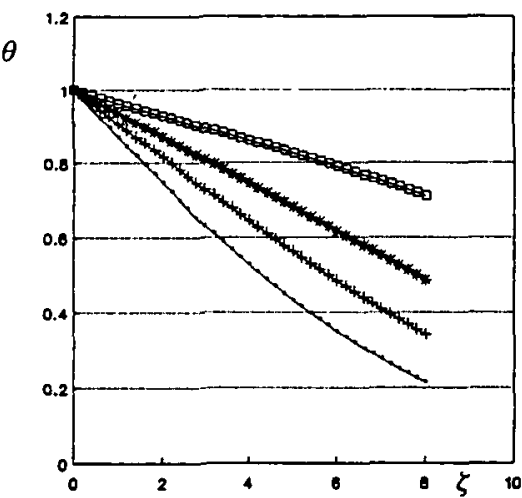

(c)

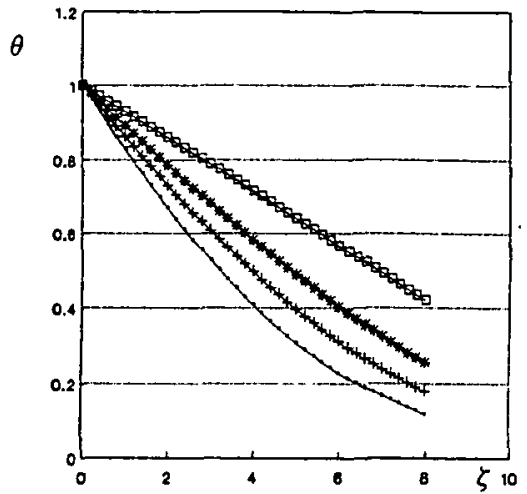

(b)

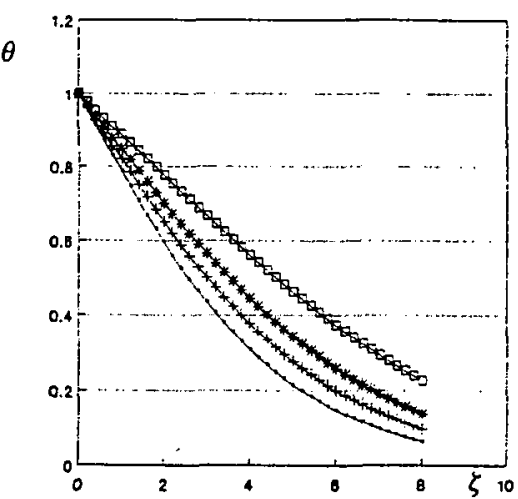

(d)

FIGURE 4. The steady-state profile of $\theta$ for various values of $K(\cdot K=0,+K=0.5, * K=1, \square K=2)$ and for (a) $\gamma=0$, (b) $\gamma=1, m=0$, (c) $\gamma=1, m=-0.5$ and (d) $\gamma=1, m=0.5$.

for higher values of $K$. Figures 1 (c) and (d) indicate that, as discussed before, a negative value of $m$ increases $G$ while a positive value of $m$ decreases it for all values of $K$.

Figure 2 presents the value of the radial velocity component $F$ as a function of $\zeta$ for various values of the parameters $K, \gamma$ and $m$. Figure 2 (a) shows that the effect of $K$ on $F$ depends on $\zeta$. Increasing $K$ decreases $F$ for small values of $\zeta$ and then increases it as $\zeta$ increases. This accounts for a crossover in the $F-\zeta$ charts for various values of $K$. Also, the effect of $K$ on $F$ is more apparent for small values of $\zeta$. Figure 2 (b) indicates interesting effects for the magnetic field in reversing the direction of $F$ for large values of $K$ and in the suppression of the crossover in the $F-\zeta$ charts with $K$. Negative values of $m$ reverse the direction of $F$ for all values of $K$ as shown in Figure 2 (c). For the Newtonian case $(K=0)$, the velocity component $F$ reverses direction beyond some 


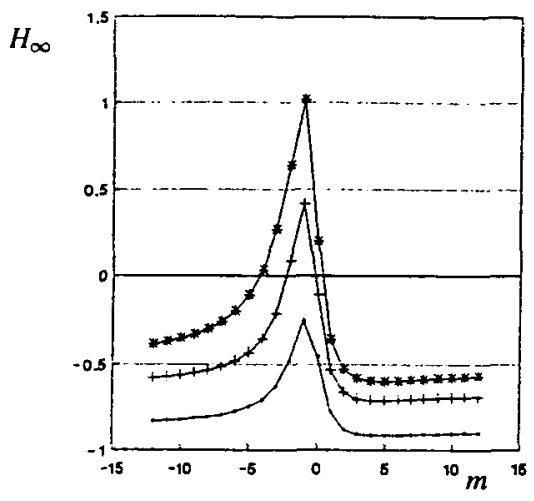

(a)

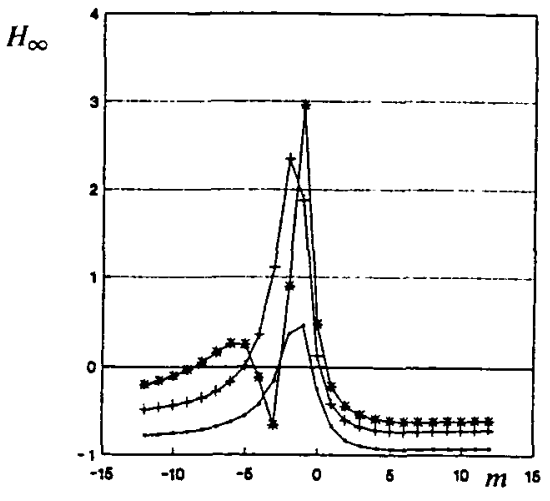

(b)

FIGURE 5. Variation of $H_{\infty}$ for various values of $m$ and $K(\cdot K=0,+K=0.5, * K=1)$ and for (a) $\gamma=0.5$ and (b) $\gamma=1$.

TABLE 1. The effect of the parameters $m$ and $K$ on $F^{\prime}(0)$.

\begin{tabular}{|l|r|r|r|}
\hline$F^{\prime}(0)$ & \multicolumn{1}{|c|}{$m=0$} & $m=-0.5$ & $m=0.5$ \\
\hline$K=0$ & 0.3093 & 0.1452 & 0.4953 \\
\hline$K=0.5$ & 0.0702 & -0.0465 & 0.2466 \\
\hline$K=1$ & -0.1109 & 0.1801 & 0.0545 \\
\hline$K=2$ & -0.3124 & -0.3172 & -0.1622 \\
\hline
\end{tabular}

distance $\zeta$ from the surface of the disk, while it reverses direction for all values of $\zeta$ in the non-Newtonian case $(K>0)$. On the other hand, positive values of $m$, as shown in Figure 2 (d), reverse the direction of $F$ only for larger values of $K(K=2)$ and small $\zeta$. Also in this case, the crossover in the $F-\zeta$ charts with $K$ appears.

Figure 3 indicates the effect of the parameters $K, \gamma$ and $m$ on the profile of the axial velocity component $H$. Increasing $K$ decreases the axial flow towards the disk (or increases $H$ ) for all values of $\gamma$ and $m$. Figure 3 (b) indicates another interesting effect of the magnetic field in reversing the direction of $H$ for large values of $K$. It is clear from Figures 3 (a) and (b) that increasing $\gamma$ decreases the axial flow towards the disk due to the damping effect of the magnetic field. Negative $m$ reverses the direction of $H$ for all $\zeta$ and $K>0$ as presented in Figure 3 (c). For the Newtonian case $(K=0)$, an intersection with the $\zeta$-axis happens and $H$ reverses direction beyond some $\zeta$. Figure 3 (d) shows that positive values of $m$ lead to a reversal in the direction of $H$ for larger values of $K$ with the presence of overshooting in $H$ with $\zeta$.

Figure 4 presents the profile of $\theta$ for various values of the parameters $k, \gamma$ and $m$ and for $\operatorname{Pr}=0.7$. The figure shows that increasing $K$ increases $\theta$ for all values of $\gamma$ and $m$ as a result of the influence of $K$ in decreasing the axial flow towards the disk which 


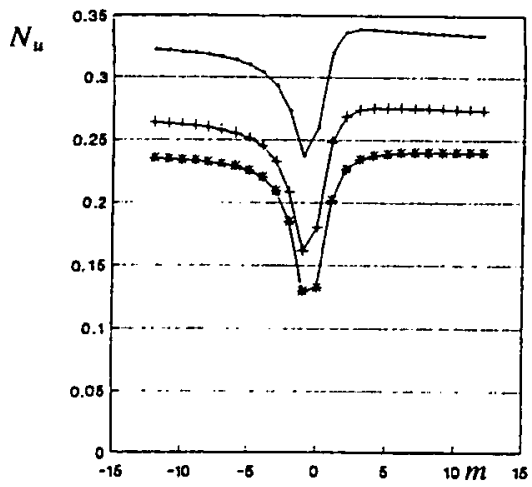

(a)

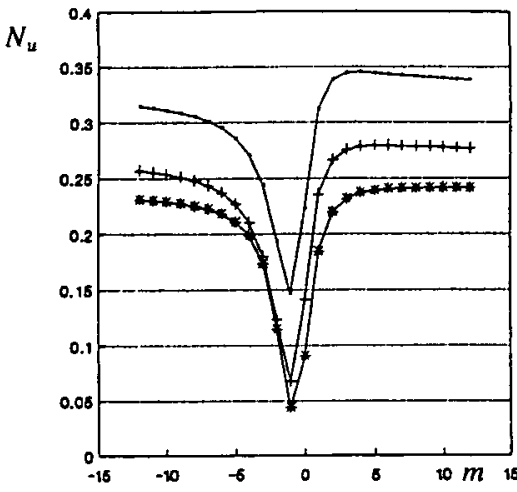

(b)

Figure 6. Variation of $N_{u}$ for various values of $m$ and $K(\cdot K=0,+K=0.5, * K=1)$ and for (a) $\gamma=0.5$ and (b) $\gamma=1$.

TABLE 2. The effect of the parameters $m$ and $K$ on $G^{\prime}(0)$.

\begin{tabular}{|l|l|r|r|}
\hline$-G^{\prime}(0)$ & $m=0$ & $m=-0.5$ & $m=0.5$ \\
\hline$K=0$ & 1.0691 & 0.9132 & 1.0626 \\
\hline$K=0.5$ & 1.0251 & 0.8531 & 1.0339 \\
\hline$K=1$ & 0.9436 & 0.7706 & 0.9628 \\
\hline$K=2$ & 0.7692 & 0.6221 & 0.7955 \\
\hline
\end{tabular}

avoids bringing the fluid at near-ambient temperature to the neighbourhood of the disk surface and consequently increases $\theta$. The effect of $K$ on $\theta$ is more pronounced for the magnetic case $(\gamma=1)$ due to the effect of the magnetic field on damping $H$. It is apparent from Figures 4 (c) and (d) that, for $\gamma=1$, negative $m$ increases $\theta$ while positive $m$ decreases $\theta$ which corresponds to the effect of the Hall parameter on the axial velocity $H$ as discussed before.

Figures 5 and 6 present, respectively, the variation of the axial velocity at infinity $H_{\infty}$ and the Nusselt number $N_{u}$ with the Hall parameter $m$ for various values of the parameters $K$ and $\gamma$ and for $\operatorname{Pr}=0.7$. For small values of $\gamma$, increasing $K$ increases $H_{\infty}$ for all values of $m$ as shown in Figure 5 (a). The peak value of $H_{\infty}$ occurs at a small and negative value of $m$ which does not depend on $K$. Also it is clear from the figure that $H_{\infty}$ reverses its direction for small values of $m$, positive or negative, and large values of $K$. For large values of $\gamma(\gamma=1)$, the variation of $H_{\infty}$ with $m$ depends on $K$. Increasing $\gamma$ reverses the direction of $H_{\infty}$ for all values of $K$. For larger values of $K$, an interesting effect of the Hall parameter in reversing the direction of $H_{\infty}$ many times is observed in Figure 5 (b). However, for small and moderate values of $K$, the peak value of $H_{\infty}$ occurs at small and negative $m$ which depends on $K$. Comparing 
Figures 5 (a) and (b) shows that increasing $\gamma$ increases $H_{\infty}$ for small $m$ while its effect is neglected for large $m$. Figure 6 shows that increasing $K$ decreases $N_{u}$ for all values of $\gamma$ and $m$. A comparison between Figures 6 (a) and (b), indicates that increasing $\gamma$ decreases $N_{u}$ for small values of $m$ and that its influence can be neglected for large positive or negative values of $m$.

Tables 1 and 2 present, respectively, the variation of $F^{\prime}(0)$ and the magnitude of $G^{\prime}(0)$ for various values of the parameters $m$ and $K$. The case $m=K=0$ corresponds to the Newtonian case in the absence of the Hall effect. Increasing $K$ decreases both $F^{\prime}(0)$ and $G^{\prime}(0)$ for all values of $m$. Higher values of $K$ may result in the reversal of the sign of $F^{\prime}(0)$ depending on $m$. Increasing $m$ decreases the magnitude of $G^{\prime}(0)$ for all $K$. Negative $m$ decreases the magnitude of $F^{\prime}(0)$ for small $K$ but increases it for higher $K$. On the other hand, positive $m$ increases the magnitude of $F^{\prime}(0)$ for small $K$ and decreases it for higher values of $K$.

\section{Conclusions}

In this paper the steady MHD flow of a non-Newtonian fluid due to the uniform rotation of an infinite disk was studied considering the Hall effect. The effects of the uniform magnetic field, the Hall current and the non-Newtonian fluid characteristics on the velocity and temperature distributions were considered. In the absence of the magnetic field, it is found that the effect of $K$ on the radial flow depends on $\zeta$ which accounts for a crossover of the $F-\zeta$ charts with $K$. The magnetic field prevents the occurrence of this crossover and leads to a reversal of the direction of the radial and axial flows for larger values of $K$ and all $\zeta$. Negative values of the Hall parameter reverse the direction of the radial and axial flow for all $\zeta$ for the non-Newtonian case $(K>0)$ and for some $\zeta$ for the Newtonian case $(K=0)$. On the other hand, for large values of $K$, positive values of the Hall parameter reverse the radial flow direction for some $\zeta$ and the axial flow direction for all $\zeta$. Changing the Hall parameter reverses the direction of the axial velocity at infinity for any value of $K$, while such a reversal occurs many times for large values of $K$. Small values of the Hall parameter have a great effect on controlling the heat transfer from the surface of the disk for all values of the parameter $K$. It is of interest to see the reversal of the sign of $F^{\prime}(0)$ for some values of the parameters $m$ and $K$.

\section{References}

[1] A. L. Aboul-Hassan and H. A. Attia, "The flow due to a rotating disk with Hall effect", Phys. Lett. A 228 (1997) 286-290. 
[2] W. F. Ames, Numerical methods in partial differential equations, 2nd ed. (Academic Press, New York, 1977).

[3] H. I. Andersson and E. de Korte, "MHD flow of a power-law fluid over a rotating disk", Eur. $J$. Mech. B Fluids 21 (2002) 317-324.

[4] H. A. Attia, "Unsteady MHD flow near a rotating porous disk with uniform suction or injection", Fluid Dyn. Res. 23 (1998) 283-290.

[5] H. A. Attia, "Transient flow of a conducting fluid with heat transfer due to an infinite rotating disk", Int. Comm. Heat Mass Transfer 28 (2001) 439-448.

[6] H. A. Attia and A. L. Aboul-Hassan, "Effect of Hall current on the unsteady MHD flow due to a rotating disk with uniform suction or injection", Appl. Math. Modelling 25 (2001) 1089-1098.

[7] E. R. Benton, "On the flow due to a rotating disk", J. Fluid Mech. 24 (1966) 781-800.

[8] W. G. Cochran, "The flow due to a rotating disk", Math. Proc. Cambridge Philos. Soc. 30 (1934) 365-375.

[9] K. Cramer and S.-I. Pai, Magnetofluid dynamics for engineers and applied physicists (McGrawHill, New York, 1973).

[10] T. M. A. El-Mistikawy and H. A. Attia, "The rotating disk flow in the presence of strong magnetic field”, in Proc. 3rd Int. Congress of Fluid Mechanics, Cairo, Egypt, 2-4 January, Volume 3, (1990), $1211-1222$.

[11] T. M. A. El-Mistikawy, H. A. Attia and A. A. Megahed, "The rotating disk flow in the presence of weak magnetic field", in Proc. 4th Conference on Theoretical and Applied Mechanics, Cairo, Egypt, 5-7 November, (1991), 69-82.

[12] G. H. Evans and R. Greif, "Forced flow near a heated rotating disk: a similarity solution", J. Fluid Mech. 22 (1988) 804-807.

[13] K. Hirose, T. Yokoyama and M. Ouchi, "Numerical study of convective heat transfer on a horizontal isothermal rotating disk", Trans. Japan Soc. Mech. Engrs., Part B 61 (1995) 3770-3775.

[14] K. Millsaps and K. Pohlhausen, "Heat transfer by laminar flow from a rotating disk", J. Aero. Sci. 19 (1952) 120-126.

[15] K. G. Mithal, "On the effects of uniform high suction on the steady flow of a non-Newtonian liquid due to a rotating disk", Quart. J. Mech. Appl. Math. 14 (1961) 401-410.

[16] G. Le Palec, "Numerical study of convective heat transfer over a rotating rough disk with uniform wall temperature", Int. Comm. Heat Mass Transfer 16 (1989) 107-113.

[17] E. M. Sparrow and J. L. Gregg, "Mass transfer, flow, and heat transfer about a rotating disk", ASME J. Heat Transfer Nov. (1960) 294-302.

[18] A. C. Srivastava, "Flow of non-Newtonian fluids at small Reynolds number between two infinite disks: one rotating and the other at rest", Quart. J. Mech. Appl. Math. 14 (1961) 353-385.

[19] G. M. Sutton and A. Sherman, Engineering magnetohydrodynamics (McGraw-Hill, New York, 1965).

[20] S. E. Tadros and F. F. Erian, "Generalized laminar heat transfer from the surface of a rotating disk", Int. J. Heat Mass Transfer 25 (1982) 1615-1660.

[21] T. von Karman, "Über laminare und turbulente Reibung", Z Angew. Math. Mech. 1 (1921) 233-235. 